\title{
Tracking and Visualizing Multi-Center Cyclones
}

\author{
Emma Nilsson*, Wito Engelke*, Anke Friederici*, Ingrid Hotz* \\ *Department of Science and Technology, Linköping University, Sweden \\ E-mail: emma.nilsson@liu.se
}
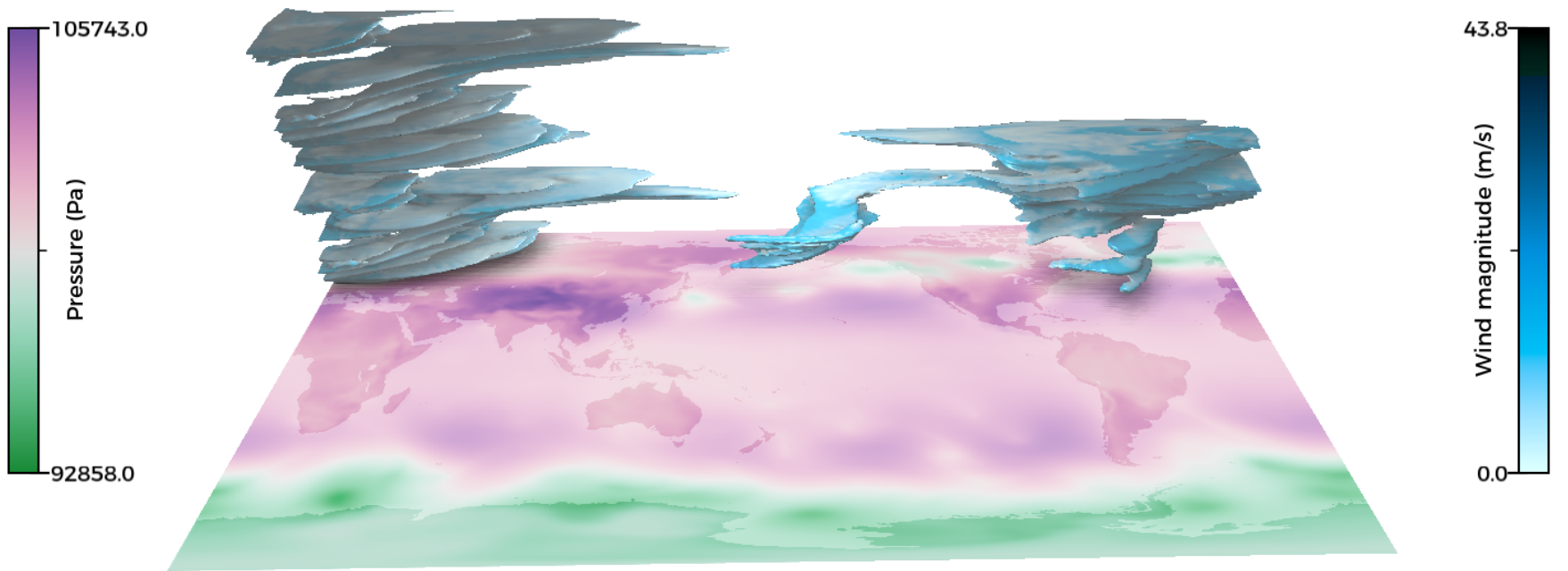

Fig. 1. An example of visualizing a chosen cyclonic feature based on our method, where the third dimension is represented by time. The cyclone track has been extracted from a tracking graph of pressure minima, while its spread and boundary is visualized using surfaces. The surface was extracted from a volume segmented using region growing, and is color mapped based on wind magnitude for a multivariate visualization.

\begin{abstract}
Cyclones are a weather phenomenon which is still actively researched today, since their complex nature make them hard to predict, track and visualize. Facilitating easy-to-use, interactive exploration and analysis of cyclones can serve as a useful tool to support domain scientists in their research. We present a framework for tracking and visualizing multicenter cyclones, which takes into account cyclones which merge and split over their lifetime. All parts of our framework allow interaction by domain scientists: the algorithm for computing the tracking graph, selections of individual cyclone tracks and the parameters used for visualizing the results. A cyclonic region defines the spread and boundary of a cyclone and over time, the pressure within the region changes. Therefore, a cyclone cannot be represented by an iso-surface in three dimensions and instead, we segment a volume by region growing from the track of the cyclone and finally, extracting a surface around the cyclonic region. We offer multiple criteria for this algorithm, allowing the domain scientist to explore and visually analyze the data. Furthermore, we enable an easy overview of the cyclone time series by mapping time to the third dimension.
\end{abstract}

Index Terms - cyclone tracking, visual exploration, weather data visualization

\section{INTRODUCTION}

Cyclones are large-scale extreme weather phenomena, with no agreed upon mathematical definition. They are characterized by high-speed rotational winds and a low-pressure center, but other weather phenomena such as precipitation are also a sign of a cyclone. Additionally, cyclones can be classified into different types: tropical cyclones are stem from tropical regions, while extra-tropical cyclones occur in midlatitude regions of the Earth. Furthermore, cyclones can have multiple centers, which can merge and split over time. These aspects make tracking and visualizing multi-center cyclones a challenging and complex task. In this paper, we present results achieved with our framework designed for solving the challenges associated with this task.

Tracking cyclones is an active research area as they are complex multivariate features. New tracking methods are continuously presented, but there are few robust methods which can be used for general cyclone tracking. Furthermore, intuitive, easy-to-use exploration of the tracks is important for domain scientists. Since tracking graphs often contain tracks which are not cyclones, but false-positives of the chosen algorithm, ways to filter and select cyclone tracks are essential. Another aspect of exploring and analyzing cyclones is being able to see their geographical spread, including the boundary of the cyclone.

The weather data used in our visualization was simulated in a two-dimensional, time-dependent domain, and is given as volumes. An illustration of the data format can be seen in Fig.2, where the boundary of the volume can be seen. The 2D spatial-components are given in longitude and latitude, while time is mapped to the third axis. To avoid clutter in the visualization, we choose to represent the globe as a map projection, where time corresponds to the third dimension, which makes it possible for the domain scientists to get an 


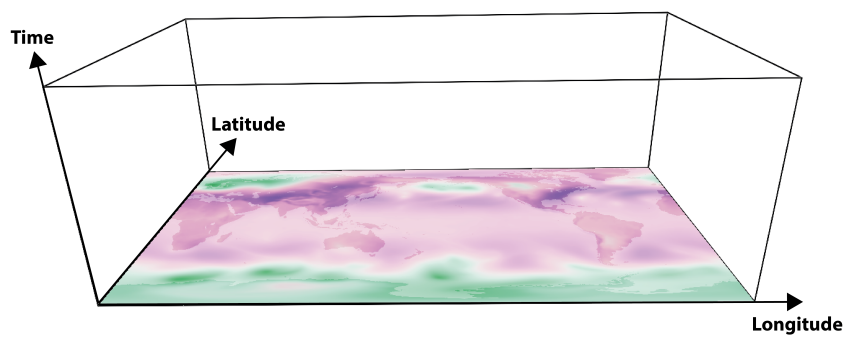

Fig. 2. The data is provided as volumes, which has a two-dimensional spatial component corresponding to longitude and latitude, while the third dimension corresponds to time. Slicing the volume with a cutting plane yields a look at the data across the entire Earth at that specific time-point.

overview of the entire cyclone.

Our cyclone tracking and visualization is based on the pressure data field and in order to not rely on a global threshold, we instead make use of a relative difference to the local pressure. This approach however prevents the use of simple scalar iso-surfaces. To combat this issue, we segment the pressure volume using region growing which extends from the cyclone tracks. From the segmented volume, we extract a final surface of the cyclonic region to visualize its extent. Furthermore, the multivariate visualization of the cyclonic region used in our framework can help domain scientists gain deeper insight into the data.

In our framework, we build on top of the robust tracking method presented by Engelke et al. [1] and facilitate filtering and selection on the raw tracking graph to maximize the flexibility of our subsequent, interactive visualization.

Our main contributions are:

- An example of applying the tracking framework presented by Engelke et al. [1] to real-world data,

- An interactive framework for filtering and selecting the input tracks,

- An interactive visualization of cyclones, which can show cyclonic regions, including their boundaries.

\section{RELATED WORK}

Many meteorological questions are related to cyclone identification, tracking, and analysis. Correspondingly, there is a lot of literature dealing with related problems, not only in the meteorology community but also in the field of visualization. In the following, we summarize the contributions that are most relevant to the work presented in this paper.

Automatic identification of cyclones from observational and numerical data has played a large role in many meteorological publications over the last 20 years [2]-[7]. A more complete list can be found in the community effort to intercompare extratropical cyclone detection and tracking algorithms [8]. Most of these methods have been developed for specific meteorological questions proposing a large variety of definitions for cyclones. They often involve a large set of parameters that have been optimized for a specific setting. The cyclone tracks mostly follow their center points that are tracked based on some heuristics based on a neighbor search. An alternative approach that tracks connected regions has been proposed by Inatsu et al. [5]. A method that inspired our tracking method is the work by Hanley et al. [7] which also considers multicentered cyclones. They achieve promising results in their application but their method is very slow and depends on several parameters and heuristics.

The cyclone tracking efforts in the visualization community try to follow more generic and efficient approaches. Thereby the common cyclone definitions have frequently been related to topological structures, considering minima, maxima, or contours of derived scalar fields. Such definitions give rise to generic topological tracking methods that are available even though often developed with other applications in mind. Examples are the critical point tracking by Reininghaus et al. [9], or contour tracking as proposed by Maadasmy et al. [10], or Lukasczyk et al. [11]. While these methods are valuable, they often cannot directly be applied without adaptation to cyclone tracking. A tracking method specifically developed for atmospheric data is the work by Doraiswamy et al. [12] As they extract tracks of clouds, however, the method is only partially applicable for the tracking of cyclones. An approach that focuses on the tracking of cyclones was presented by Valsangkar et al. [13]. Their work is also one of the few methods that provide some limited exploration options.

Even though these approaches work well in one setting they often miss the necessary flexibility to be useful in a larger context. This is partially because cyclones are not mathematically well-defined objects and the domain scientist intuition cannot easily be integrated into existing methods. Especially the use of global parameters for the definition of cyclones does not take the global variations of pressure into account. Engelke et al. [1] have presented a method that can cope with this challenge. They extract a raw topological tracking graph as a basis for domain-specific feature definitions and feature tracks. Their work is the basis for the visualizations presented in this paper. A typical challenge when visualizing temporal trajectories using an embedding in space, e.g. overlaid over a map, is the fact that they may overlap, which results in cluttered visualizations. In the context of tracking ash clouds, Kuhn et al. [14] avoid this problem by using the third dimension of the visualization space to represent time. This is a concept that we also use in our work.

Cyclones are often visualized by only using the wind data field, and in scientific visualization virtual globes is a common Earth representation. In the work by Wang et al. [15], they visualize polar cyclones by only representing the wind field with particles, even though they use pressure to track the cyclones. The spread of the cyclones can be visually identified by finding the rotating parts of the wind field, but they do not define a strict boundary. Another example of visualizing cyclones using virtual globes is the work by Liu et al. [16], where the user can choose whether to visualize the wind using flow arrows, streamlines or particles. 


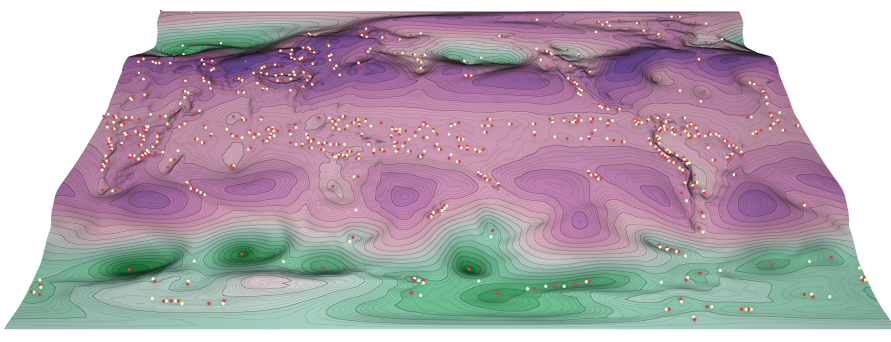

(a) Pressure Field

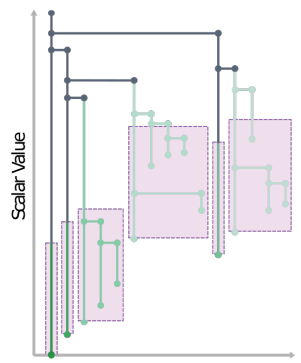

(b) MT Branches

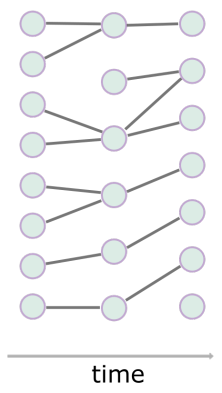

(c) Tracking of CPs

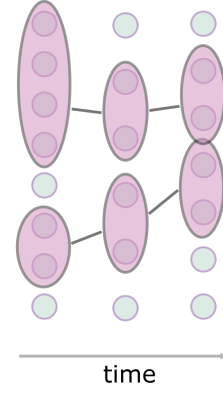

(d) Sets of CPs

Fig. 3. Tracking Pipeline: The pipeline for the cyclone tracking, starting with the extraction of all extremal points (see (a) showing minima and saddles) in the pressure field. (b) The branch decomposition of a merge tree is used to identify relevant minima (c) The raw tracking graph containing tracks of minima (d) Cyclones are defined as sets of critical points. This allows for merges, splits, birth and death.

\section{METHOD}

In this section we present the three parts of our framework: 1. cyclone tracking, 2. selecting and filtering cyclone tracks and 3 . visualizing cyclonic regions.

\section{A. Cyclone identification and tracking}

The cyclone exploration presented in this work builds on a cyclone-tracking method that will be briefly introduced in this section. The method is based on two independent steps. First is the identification of cyclones based on sets of critical points and subsequent the computation of point to point correspondence between time steps. This information is stored in the raw tracking graph. Cyclone tracks are generated by combining both steps. In detail the individual steps are:

Cyclone identification: A cyclone is represented as a set of critical points (CP) of a selected scalar field used as an identifier for cyclones, compare Figure 3. Typical examples are the minima in a pressure field or maxima of vorticity magnitude. The sets of CPs are combined according to a few simple rules facilitating a merge tree of the selected scalar field [17]. The rules can be designed by the domain scientists and they are independent of the tracking method used in the subsequent steps.

Raw tracking graph: The raw tracking graph stores information about the point-to-point correspondence as directed edges between the critical points, represented as vertices. For this information the contribution of an individual critical point to a cyclone is not considered. The correspondence information is calculated based on the topological concepts of feature flow fields, using a Morse matching based on the Morse decomposition of the scalar field [9]. In this graph, every $\mathrm{CP}$ has exactly one successor and predecessor. However, one critical point can share a successor with another critical point. The result of this step is a large directed graph containing all available information of point-based temporal correspondence with respect to a Morse decomposition of the domain.

Cyclone tracking: Tracking of cyclones is based on the tracking of individual critical points. Since the set of rules used for cyclone identification is independent from the information stored in the tracking graph cyclone tracking is realized as queering the graph. This is illustrated in Figure 3. CPs that are not part of a cyclone are not considered. The resulting cyclone tracks allow for birth, death, merge and split events of cyclones. Additionally it also supports multi-centered cyclones represented by disconnected contours. Separating the two steps gives the domain scientist the possibility to change the rules of cyclone identification without requiring a new computation of the tracking, the most expensive step of the method.

\section{B. Selecting cyclone tracks}

The tracking graph algorithm results in a graph of $\mathrm{CP}$ on a two-dimensional map over time, which are all connected. The resulting cluttered tracking graph is visualized in Figure 6(a). Notably, all connections in the graph do not represent cyclones and are therefore not physically meaningful for the domain scientists. To facilitate insights from this large amount of information, one part of our presented interactive framework is the option to filter and select which part of the tracking graph is given as input to the visualization.

The tracking graph can be filtered based on spatial position and edge length. Edge lengths can either be filtered on the Euclidean distance in Cartesian space, or the great circle distance. Filtering based on the Euclidean distance corresponds to the edge length the user sees in the framework, while the great circle distance corresponds to a correct geographical edge length, which is not shown directly.

The great circle distance is calculated by using the Haversine formula, which is shown in (1). In the formula, the haversine of the central angle $\Delta \sigma$ is expressed using the haversine function $\operatorname{hav}(\theta)=\sin ^{2}\left(\frac{\theta}{2}\right)$, in which $\theta$ is the angle. The formula uses the latitudes $\phi_{1}$ and $\phi_{2}$ and the longitudes $\lambda_{1}$ and $\lambda_{2}$ of the points to get the haversine of the central angle.

$$
\operatorname{hav}(\Delta \sigma)=\operatorname{hav}\left(\phi_{2}-\phi_{1}\right)+\cos \left(\phi_{1}\right) \cdot \cos \left(\phi_{2}\right) \cdot \operatorname{hav}\left(\lambda_{2}-\lambda_{1}\right)
$$

In (2), the haversine of the central angle given by the haversine formula is used to calculate the great circle distance $d$ between two points. 


$$
d=2 \cdot r \arctan \left(\frac{\operatorname{hav}(\Delta \sigma)}{1-\operatorname{hav}(\Delta \sigma)}\right)
$$

\section{Visualizing cyclones}

The weather data is simulated over time in a twodimensional map-based domain, and we choose to map time to the third dimension in our visualization. Consequently, domain scientists can quickly overview entire cyclonic regions, in contrast to a virtual globe representation, where the entire Earth cannot be shown at once.

The cyclone tracks selected visualize the movement of the cyclone's eye/s, but do not show how a cyclone is spread or how that spread changes over time. Visualizing the spread and consequently the cyclone's boundary is a complex task. Instead of a global threshold, we rely on a moving pressure window around the current cyclone pressure minimum describing its center. This effectively allows us to view each cyclone region in its own context regardless of the surrounding conditions. The spread can be defined by the difference in pressure allowed between the eye and the surrounding region in the pressure field. Consequently, the boundary of the cyclone is the maximum allowed difference in pressure.

Commonly, regions of matching values are visualized with iso-surfaces in a 3D domain. However, this technique cannot be immediately applied here, since it can only extract regions of matching values, not regions in which values vary. For cyclones, the pressure varies in the eye, the region around it and on its' boundary. As mentioned earlier, cyclones can have multiple eyes, which can split and merge during the movement of its trajectory. Accordingly, any surface extraction algorithm used to extract the cyclonic region needs to be able to handle these cases.

In this work, the pressure volume is segmented using region growing, generating a new volume from which a surface can be extracted using Marching cubes [18]. There are other possible techniques, such as slicing the volume, extract iso-contours and manually connect the contours together over time, but this method was chosen for its simplicity and efficiency.

The region growing algorithm used requires seeds. They set what pressure values should be used a a reference, as well as from which spatial positions in the volume the region should be grown. In this work, the tracks of the cyclone's eyes are used to that end. The region growing algorithm takes the voxel containing the eye, adding in all neighboring cells fulfilling a selection criterion.

These criteria define the requirements for a voxel to belong to the cyclonic region. In this work, the user can pick between four criteria. They highlight different features describing a cyclone, for example allowing to consider strong pressure gradients as indicators of a cyclone's size. The first criterion is in (3), in which $v$ is the pressure value of the voxel being processed, $v_{s}$ the pressure value in the cyclone's eye and $b$ is the user-defined boundary. The boundary defines the maximum allowed pressure difference between the eye and the cyclonic region.

$$
\left|v-v_{s}\right|<b
$$

The other three criteria implemented are all from the paper by Huang et al. [19], and they use statistical measures of the neighbouring voxels to the seed voxels in their criteria. One criterion is based directly on voxel values, one on the gradient magnitude of the neighbouring voxels while the third criterion is a mix of the previous two. The first criterion from the paper is defined in (4), in which $v$ is the pressure in the current voxel, $v_{s}$ the pressure in the seed voxel, $k$ a user-defined constant and $\sigma_{v}$ the standard deviation of the pressure in the surrounding 26 neighbour voxels to the seed voxel. This criterion finds homogeneous areas in the volume, and the user can set how strict it is by changing $k$. A smaller $k$ makes the allowed pressure difference in the region less, while a larger $\mathrm{k}$ allows a larger difference in pressure.

$$
\left(f_{c a}=\frac{\left|v-v_{s}\right|}{k \cdot \sigma_{v}}\right)<1
$$

The second criterion $f_{c b}$, see (5), uses the gradient magnitude of the pressure in the voxels. In (5), $g$ and $g_{s}$ is the gradient magnitude of the current voxel and seed voxel respectively, while $k$ is still a user-defined constant and $\sigma_{g}$ is the standard deviation of the gradient magnitude of the neighbouring 26 voxels to the seed voxel. Using the gradient magnitude allows easily identifying edges in the volume, which can help finding the boundaries of cyclonic regions.

$$
\left(f_{c b}=\frac{\left|g-g_{s}\right|}{k \cdot \sigma_{g}}\right)<1
$$

The third criterion $f_{c c}$ in (6) weights the two previous criteria together. The user-defined coefficient $p$ decides the weighted sum, and a larger $p$ weights more of the first criterion while a smaller $p$ uses more of the second.

$$
\left(f_{c c}=p \cdot f_{c a}+(1-p) \cdot f_{c b}\right)<1
$$

If the voxel fulfills the criterion chosen by the user, the process is as follows: 1. the voxel is added to the queue, and 2 . in the created volume, the corresponding voxel is set to the value calculated by the criterion. If the voxel does not fulfill the criterion, the corresponding voxel in the output volume is still set to the calculated value, but the voxel is not added to the queue. When all neighbouring voxels have gone through the process, the next voxel is taken from the queue and the same method is applied over again until the queue is empty. When the queue is empty, the algorithm is applied to the next seed voxel until there are no left.

The result is a segmented volume, in which only voxels belonging to or neighbouring the cyclonic region have a value. The value range of the cyclonic region in the volume depends on what criterion is chosen by the user: criterion (3) gives a value between $[0, b]$ while the three criteria defined by Huang et al. [19] results in a value range between $[0,1]$. The final surface is generated by applying Marching cubes [18], where 


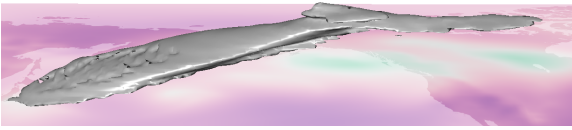

(a) Time-slice 23

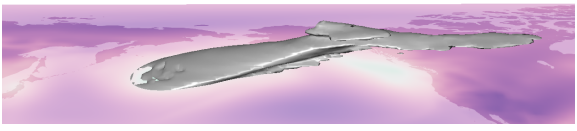

(b) Time-slice 34

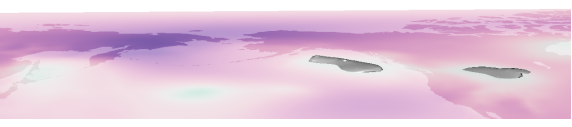

(c) Time-slice 51

Fig. 4. Surface Extraction: A surface generated using the boundary criterion (3) and the cyclone track in Figure 7, shown at three different time-slices. The time-slices are color mapped with the pressure of the field at that time-point, where green is low pressure and purple is high pressure. The surface covers a connected, low pressure region, which over time moves across the Northern Pacific Ocean from Japan's east coast towards the west coast of the United States. Just before the cyclonic region disappears, it is split into two individual regions, showing that the surfaces generated can handle merge and split events.

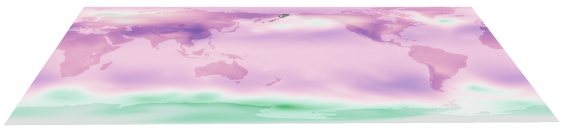

(a) $k=0.1$

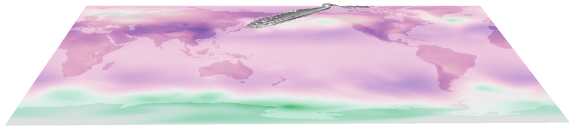

(b) $k=0.5$

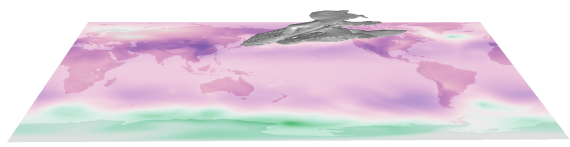

(c) $k=1.0$

Fig. 5. Surface Extraction: Examples of surfaces generated using the criterion in Equation (4) and the cyclone track in Figure 7. The parameter $k$ is varied for the different surfaces, and a larger $k$ yields a larger surface. Beneath the surfaces is a quad, which shows the pressure variation in the field. As before, green represents low pressure while purple visualizes high pressure.

the iso-value is set to either $b$ or 1 , depending on the criterion chosen.

\section{RESUlTs}

The results presented in this section were generated from the ERA Interim data set from the European Centre for MediumRange Weather Forecasts (ECMWF). The data is simulated every 6:th hour, but for these results we have restricted ourselves to one month, December 1999. The data contains a global volumetric pressure field, which is two-dimensional and time-dependent, where the third dimension corresponds to time. In the following section, a time-slice is a cutting plane of the volume. The time spans 248 slices, which each represents 6 hours of the simulation.

An example of filtering cyclone tracks can be seen in Fig.6, in which (a) shows the unfiltered tracking graph, while (b) shows the result of filtering away all vertices between $-30^{\circ}$ and $30^{\circ}$ latitude. The quad beneath the graph shows a world map and the pressure at time-slice zero. The quad can be moved to slice the volume at different time-points, which visualizes which cyclone tracks are in low pressure regions. While this filtering is useful, using these amount of tracks will yield a surface which covers the entire volume. To get a surface of one low pressure area, a small part of the graph must be selected.

In Fig.7, a miniscule track from the graph is shown. The track is in the Northern Pacific Ocean, and spans time-slices 28 to 35 . The track has merging and splitting events, and resides in a low pressure area. The track was selected by exploring the tracking graph using filtering in combination with looking at low pressure areas in different time-slices.

The selected cyclone track from Fig.7 is used to generate the surface shown in Fig.4. The surface of the low pressure, cyclonic region is shown in three individual time-slices: 23 , 34 and 51. It was generated by using the user-set boundary criterion in (3), where $b$ was set to 1000 . Consequently, the cyclonic region allows a $1000 \mathrm{~Pa}$ difference between the cyclone's eye/s and the boundary of the cyclone. As shown in the figure, the surface generated spans more time-slices than the track does, and we cannot see the merging and splitting events from the track in the surface. However, it is still possible for the surface algorithm to handle a low pressure region splitting into two, see Fig.4(c), where the surface is no longer connected, but still covers two low pressure areas.

It is also of interest to see how the surface changes using different values for the parameters in the criteria. While this is intuitive for the user-set boundary criterion in (3), it is not as clear how varying $k$ or $p$ affects the resulting surface. In Fig.5, all surfaces have been created using the criterion in Equation (4) and the cyclone track in Fig.7. As mentioned earlier, this criterion aims at finding homogeneous regions in the volume. For the different images in the figure, $k$ has been varied, and we can see that a smaller $k$ yields a smaller surface, while a larger $k$ results in a larger surface. This is because a larger $k$ allows a larger difference in pressure for a voxel to be included in the region.

In contrast to the small regions grown using criterion (4), using similar $k$ in criterion (5) yields enormous surfaces. This is exemplified in Fig.8. The pressure volume contains many edges, and since the criterion aims at finding boundaries in the volume, using the criterion yields large surfaces. When $k$ is set to 1.0 , the entire volume is encased in a surface and even at $k=0.5$, the surface covers multiple regions in the volume.

Creating surfaces by using criterion (6) mixes the two previous criteria together, consequently both homogeneous regions and edges should be captured by the criterion. The results from using this criterion when region growing, along with the cyclone track in Fig.7, is shown in Fig.9. In the top row of the figure, $k$ is varied while $p$ is set to 0.5. Setting $p$ to 0.5 results in both criteria being equally weighted in the region growing. Compared to Fig.5 and Fig.8, the surfaces 


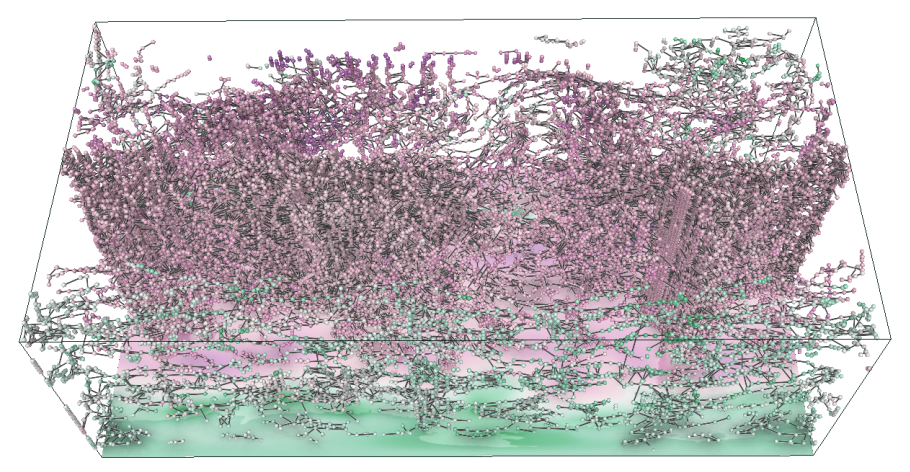

(a)

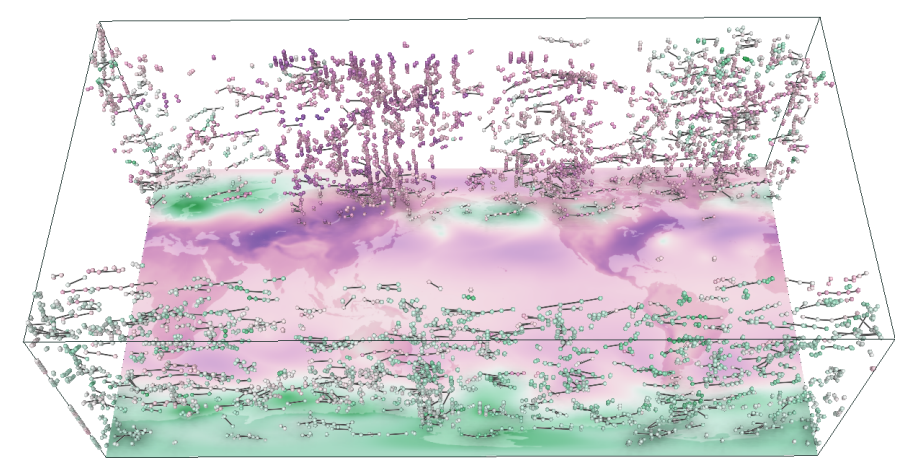

(b)

Fig. 6. Raw Tracking Graph:An example of the visual difference between filtering vs. not filtering cyclone tracks. In figure (b), all vertices between $-50^{\circ}$ and $50^{\circ}$ latitude and all edges longer than $5000 \mathrm{~km}$ have been removed from the tracking graph. The underlying quad visualizes the pressure of the field at time-slice 0 , in which green is low pressure and purple is high pressure. The vertices are also color-mapped with the pressure field.

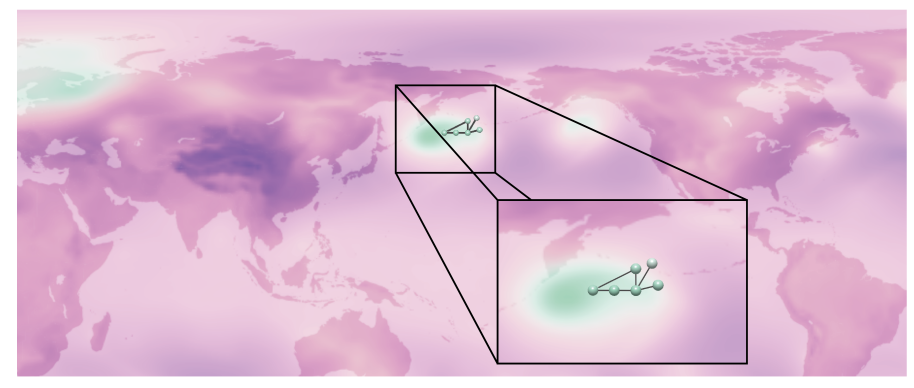

Fig. 7. Selection: A small track selected from the tracking graph, spanning time-slices 28-35 with its position in the Northern Pacific Ocean. The track has merging and splitting events and exists in pressure minima. The vertices of the track underlying time-slice are color mapped, where green signifies low pressure and purple signifies high pressure.

created with this weighting are smaller and more compact. Here, increasing $k$ still yields larger surfaces. In the bottom row, $p$ is varied, while $k$ is set to 1.0. A smaller $p$ takes more of the edge criterion in (5) into account, consequently the surface is large with multiple holes, since we mostly look at the edges between regions. When $p$ instead is set to 0.9 , we search for homogeneous regions, which gives a smaller, more compact surface. Even so, this surface still has holes on its edges. Comparing these two surfaces to the last image in the top row, which also has $k=1.0$ while $p$ is set to 0.5 , they are much larger, but less compact.

The results show that depending on criterion chosen, the defined cyclonic feature will vary greatly. Criterion (3) is the most intuitive for the user, since $b$ is the pressure difference allowed, while criteria (4) - (6) allow the user to change $k$, which does not have a similar connotation. However, $k$ does change the allowed pressure difference just like $b$, but it is not given in Pascal, which may be confusing. For criterion (4), setting $k$ to 1 will lead to comparing voxel values with the standard deviation around the cyclone track, which includes information the user cannot see in the framework. This may lead - initially - to quicker extraction of interesting regions, while setting an explicit boundary can take some more experimentation before the wanted feature is found.

\section{DisCUSSION}

With the proposed interactive framework, it is possible for the user to select individual cyclone tracks and extract a surface of the cyclone's boundary. Furthermore, the user can also be in control of how the surface is extracted by using different criteria and parameters. Even so, there are still multiple improvements that could be made.

Firstly, the cyclone tracks can only be filtered based on spatial position and edge length. To find cyclone tracks in low pressure regions, the user must use the time-slice quad to see the pressure of the region the cyclone track is in. As a consequence, it is a tedious process to move through time-slices and see where the region starts and ends, furthermore what cyclone track to select. An improvement to the framework is including a filtering option where the user can threshold the graph based on a pressure value. Thus, the user can quickly see the cyclone tracks in low pressure regions and select the appropriate ones to feed to the region growing algorithm.

Secondly, a cyclone is only defined by its pressure in the framework, which is in contrast to cyclones multivariate nature. Taking multiple variables into account when tracking may give tracks which better fit real-world cyclones. Furthermore, visualizing the cyclones using multivariate visualization could improve the visual analysis of them for the domain scientists. Visualizing fields which also signifies cyclones, such as wind and temperature, and how these change within the region and on the boundary, could improve the data exploration experience and give deeper insight on the data for the domain scientists. Including a multivariate visualization in future work would be an improvement on the current framework.

Regarding the chosen surface extraction method, a benefit is the previously mentioned control the user has over the extracted surface. It is also notable that the selected cyclone track greatly affects the result of the region growing, as the track contains the seeds given to the algorithm. Therefore, if the 


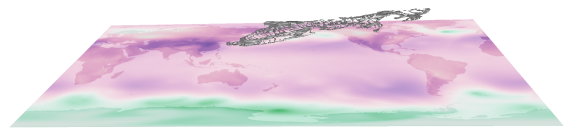

(a) $k=0.1$

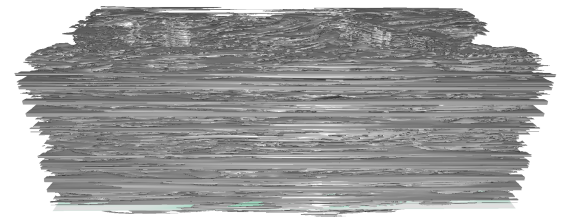

(b) $k=0.5$

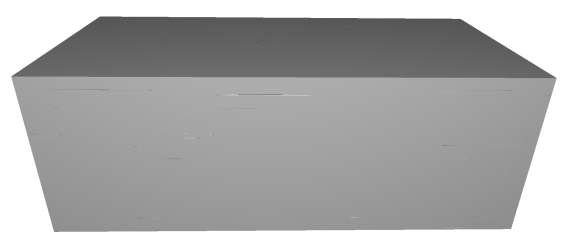

(c) $k=1.0$

Fig. 8. Surface Extraction: Examples of surfaces generated using the criterion in Equation (5) and the cyclone track in Figure 7. The parameter $k$ is varied for the different surfaces, and here as $k$ grows, the entire volume is encased in a surface. Once more, the underlying quad shows the pressure, where green regions represent low pressure areas and purple regions high pressure areas.

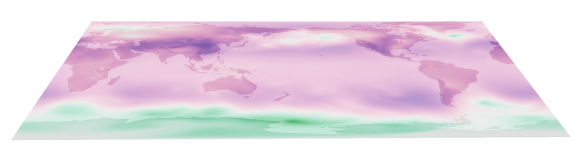

(a) $k=0.1, p=0.5$

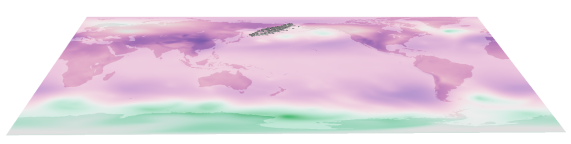

(b) $k=0.5, p=0.5$

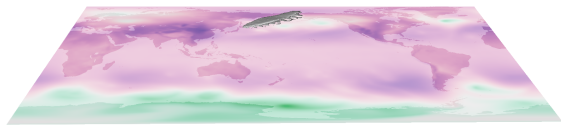

(c) $k=1.0, p=0.5$

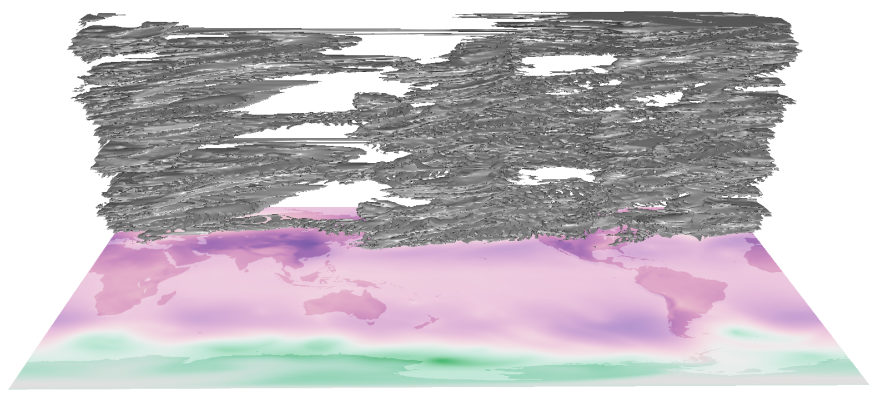

(d) $k=1.0, p=0.1$

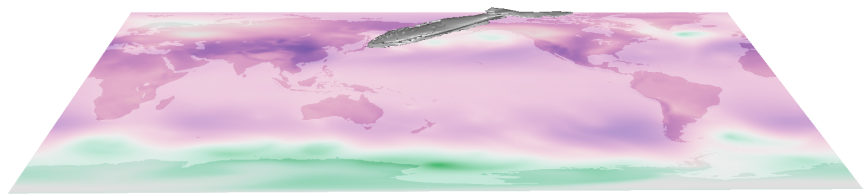

(e) $k=1.0, p=0.9$

Fig. 9. Surface Extraction: Examples of generating surfaces by using the criterion in Equation (6) and the cyclone track in Figure 7. In the top row of the image, the weighting variable $p$ is set to 0.5 while $k$ is varied. In the bottom row, $p$ is varied while $k$ is set to 1.0. A larger $k$ yields larger surfaces, while a larger $p$ weights more of the criterion in Equation (4) and therefore yields a smaller surface than a smaller $p$ would. The underlying time-slices from the volume are color mapped with the pressure, where green shows low values and purple shows high values.

user accidentally selects a track where the pressure difference is large, the surface generated can quickly encase the entire volume. Consequently, the exploration of the cyclone tracks is essential to get a surface which represents the boundary of the cyclone, rather than all similar regions in the volume.

The region growing could also be done by taking multiple variables into account, such as temperature or the wind velocity. Currently, by only using the pressure, all low pressure regions containing tracks from the tracking graph can be considered cyclonic regions. By forcing more restrictive criteria, it may be easier to find actual cyclonic regions, and not just low-pressure regions. Future work in this area could simplify the exploration of the data set for the domain scientists.

\section{CONCLUSION}

Tracking and visualizing multi-center cyclones is a complex problem. We have presented a framework for tracking cy- clones, selecting individual cyclone tracks and visualizing the cyclonic region, including its boundary. With our framework, the domain scientists can visually explore and analyze the data, but we conclude that the filtering and selecting of the cyclone tracks require more options for real-world scenarios. In future work, a multivariate definition of cyclones could be used to achieve more realistic cyclonic regions and boundaries. Furthermore, a multivariate visualization would help domain scientists gain deeper insight on the data.

\section{ACKNOWLEDGMENT}

For implementation the visualization-framework Inviwo [20] was used. This work was supported through grants from the Excellence Center at Linköping and Lund in Information Technology (ELLIIT) and the Swedish e-Science Research Centre (SeRC) and the grant from the Swedish Foundation for Strategic Research (SSF, BD15-0082). 


\section{REFERENCES}

[1] W. Engelke, J. Beran, R. Caballero, T. B. Masood, and I. Hotz, "Topology-based feature design and tracking for multi-center cyclones," in Topological Methods in Data Analysis and Visualization, I. Hotz, T. B. Masood, F. Sadlo, and J. Tierny, Eds., no. 9. Springer, to appear 2021.

[2] R. J. Murray and I. Simmonds, "A numerical scheme for tracking cyclone centres from digital data," Australian Meteorological Magazine, vol. 39, no. 3, 1991 .

[3] K. I. Hodges, "A General Method for Tracking Analysis and Its Application to Meteorological Data," Monthly Weather Review, vol. 122, no. 11 , pp. 2573-2586, 1994.

[4] M. R. Sinclair, "Objective Identification of Cyclones and Their Circulation Intensity, and Climatology," Weather and Forecasting, vol. 12, no. 3, pp. 595-612, 1997.

[5] M. Inatsu, "The neighbor enclosed area tracking algorithm for extratropical wintertime cyclones," Atmospheric Science Letters, vol. 10, no. 4, pp. 267-272, 2009.

[6] U. Ulbrich, G. Leckebusch, and J. G. Pinto, "Extra-tropical cyclones in the present and future climate: a review," Theoretical and Applied Climatology, vol. 96, no. 1-2, pp. 117-131, 2009. [Online]. Available: https://doi.org/10.1007/s00704-008-0083-8

[7] J. Hanley and R. Caballero, "Objective identification and tracking of multicentre cyclones in the era-interim reanalysis dataset." Quarterly Journal of the Royal Meteorological Society, pp. 612-625, 2012.

[8] U. Neu, M. G. Akperov, N. Bellenbaum, R. Benestad, R. Blender, R. Caballero, A. Cocazza, H. F. Dacre, Y. Feng, K. Fraedrich, J. Grieger, S. Gulev, J. Hanley, T. Hewson, M. Inatsu, K. Keay, S. F. Kew, I. Kindem, G. C. Leckebusch, M. L. R. Liberato, P. Lionello, I. I. Mokhov, J. G. Pinto, C. C. Raible, M. Reale, I. Rudeva, M. Schuster, I. Simmonds, M. Sinclair, M. Sprenger, N. D. Tilinina, I. F. Trigo, S. Ulbrich, U. Ulbrich, X. L. Wang, and H. Wernli, "Imilast: A community effort to intercompare extratropical cyclone detection and tracking algorithms," American Meterorological Society (AMS) Journal, pp. 529-549, 2013.

[9] J. Reininghaus, J. Kasten, T. Weinkauf, and I. Hotz, "Efficient computation of combinatorial feature flow fields," IEEE Transactions on Visualization and Computer Graphics, vol. 18, no. 9, pp. 1563-1573, 2012.

[10] S. Maadasamy, H. Doraiswamy, and V. Natarajan, "A hybrid parallel algorithm for computing and tracking level set topology," 20th Annual International Conference on High Performance Computing, vol. 0, pp. 1-10, 2012.

[11] J. Lukasczyk, G. Weber, R. Maciejewski, C. Garth, and H. Leitte, "Nested tracking graphs," Computer Graphics Forum, vol. 36, no. 3, pp. 13-22, 2017.

[12] H. Doraiswamy, V. Natarajan, and R. S. Nanjundiah, "An exploration framework to identify and track movement of cloud systems," IEEE Transactions on Visualization and Computer Graphics (Vis'13), vol. 19, no. 12, pp. 2896-2905, 2013.

[13] A. A. Valsangkar, J. M. Monteiro, V. Narayanan, I. Hotz, and V. Natarajan, "An exploratory framework for cyclone identification and tracking," IEEE Transaction on Visualization and Computer Graphics, vol. 25, no. 3, pp. 1460-1473, 2018.

[14] A. Kuhn, W. Engelke, M. Flatken, H.-C. Hege, and I. Hotz, "Topologybased analysis for multimodal atmospheric data of volcano eruptions," in Topological Methods in Data Analysis and Visualization IV, Theory, Algorithms, and applications, ser. Mathematics and Visualization, H. Carr, C. Garth, and T. Weinkauf, Eds. Springer, 2017.

[15] F. Wang, W. Li, and S. Wang, "Polar cyclone identification from 4d climate data in a knowledge-driven visualization system," Climate, vol. 4, no. 3, p. 43, 2016.

[16] P. Liu, J. Gong and M. Yu, "Visualizing and analyzing dynamic meteorological data with virtual globes: A case study of tropical cyclones," Environmental Modelling \& Software, pp. 80-93, 2015.

[17] H. Carr, J. Snoeyink, and U. Axen, "Computing contour trees in all dimensions," Computational Geometry, vol. 24, no. 2, pp. 75-94, 2000.

[18] William E. Lorensen and Harvey E. Cline, "Marching cubes: A high resolution 3d surface construction algorithm," ACM SIGGRAPH Computer Graphics, pp. 163-169, 1987.

[19] R. Huang and K.-L. Ma, "Rgvis: Region growing based techniques for volume visualization," in 11th Pacific Conference on Computer Graphics and Applications, 2003. Proceedings. IEEE, 2003, pp. 355-363.
[20] D. Jönsson, P. Steneteg, E. Sundén, R. Englund, S. Kottravel, M. Falk, A. Ynnerman, I. Hotz and T. Ropinski, "Inviwo - a visualization system with usage abstraction levels," IEEE Transactions on Visualization and Computer Graphics, 2019. 